\title{
Peptide Conformation in Gas Phase Probed by Collision-Induced Dissociation and Its Correlation to Conformation in Condensed Phases
}

\author{
Zhongqi Zhang and Joseph Bordas-Nagy \\ Amgen Inc., Thousand Oaks, California, USA
}

\begin{abstract}
A kinetic peptide fragmentation model for quantitative prediction of peptide CID spectra in an ion trap mass spectrometer has been reported recently. When applying the model to predict the CID spectra of large peptides, it was often found that the predicted spectra differed significantly from their experimental spectra, presumably due to noncovalent interactions in these large polypeptides, which are not considered in the fragmentation model. As a result, site-specific quantitative information correlated to the secondary/tertiary structure of an ionized peptide may be extracted from its CID spectrum. To extract this information, the kinetic peptide fragmentation model was modified by incorporating conformation-related parameters. These parameters are optimized for best fit between the predicted and the experimental spectrum. A conformational stability map is then generated from these conformation-related parameters. Analysis of a few bioactive $\alpha$-helical peptides including melittin, glucagon and neuropeptide $Y$ by this technique demonstrated that their stability maps in the gas phase correlate strongly to their secondary structures in the condensed phases. (J Am Soc Mass Spectrom 2006, 17, 786-794) (c) 2006 American Society for Mass Spectrometry
\end{abstract}

\section{$\mathrm{H}$} Tow a one-dimensional polypeptide chain folds into a three-dimensional biologically active form is one of the greatest challenges in life science. One approach to understand the role of solvent in this folding process is to study protein conformation in the gas phase, in the absence of solvent. Understanding the relationship between the gas-phase and condensed-phase protein conformations will also help us evaluate mass spectrometry as a tool for studying protein noncovalent interactions.

Protein conformation and dynamics in the condensed phases has traditionally been studied by X-ray crystallography [1], nuclear magnetic resonance spectroscopy [2-4], circular dichroism [5], etc., as well as hydrogen exchange $[6,7]$ and its recent combination with mass spectrometry [8-11]. Compared to the large amount of knowledge about protein conformation in the condensed phases, little is known about the conformation of proteins in gas phase, largely due to the lack of structurally informative techniques to study gasphase proteins $[12,13]$. Techniques including collision cross-section measurement [14-21] and hydrogen/deuterium exchange [22-27] have been used to study protein gas-phase conformation, but they provide limited structural details. Recently, electron-capture dissocia-

Published online April 3, 2006

Address reprint requests to Dr. Z. Zhang, Amgen Inc., 30E-1-B, One Amgen Center Drive, Thousand Oaks, CA 91320, USA. E-mail: zzhang@amgen.com tion (ECD) combined with Fourier-transform ion cyclotron resonance mass spectrometry shed some light on the structural details of gaseous proteins [28-30]. This paper reports a new methodology, based on collisioninduced dissociation (CID) spectra of peptides or proteins, for structural details of gaseous peptides or proteins that compliments the information obtained from ECD and other conventional techniques. Smith and coworkers first demonstrated that CID spectra could be used as a probe for protein conformation [31]. Cassady and Carr also used CID to probe conformation of ubiquitin ions [24]. However, the information derived from these experiments was largely qualitative. To derive conformation-related information from protein/ peptide CID spectra that is more quantitative in nature, the effects of primary sequence to the CID spectra need to be quantitatively removed.

A kinetic peptide fragmentation model for simulating peptide CID spectra in an ion trap mass spectrometer has been reported recently [32, 33]. The model contains parameters describing the kinetics of the fragmentation processes during a CID experiment, such as activation energy (Ea) and gas-phase basicity (GB) associated with each amino acid residue. In the model, the distribution of charges is first calculated according to GB of each protonation site. The rate constant for each fragmentation pathway is then calculated, using Arrhenius equation, based on Ea and pre-exponential frequency factor for the specific pathway and cleavage 
site. The abundance of each product ion is calculated based on the charge distribution and fragmentation rate constants for all competing pathways.

The kinetic model has been shown quite accurate in predicting peptide CID spectra, especially for relatively small peptides, and has been successfully used for reliable de novo sequencing [34]. For large peptides or proteins, however, the predicted spectra often differed significantly from their experimental spectra, presumably due to noncovalent interactions in these large polypeptides, which are not considered in the fragmentation model. If this is indeed the underlying cause for the observed discrepancies between predicted and experimental CID spectra, then site-specific quantitative information correlated to the secondary/tertiary structure of an ionized peptide may be extracted from its CID spectrum. We found that it is indeed possible to generate a stability map for a gas-phase peptide ion using this approach. Analysis of a few bioactive $\alpha$-helical peptides by this technique demonstrated that the stability maps of these polypeptides in the gas phase correlate strongly to their secondary structures in the condensed phases.

\section{Computational Method}

The noncovalent structure of a peptide is assumed to affect its fragmentation spectrum by the following mechanisms (1). Peptide backbone cleavages involve a rearrangement of the peptide conformation near the cleavage site [35-37]. To facilitate this conformational rearrangement, the conformation near the cleavage site must be flexible. Therefore, when noncovalent interactions are present near a backbone cleavage site, an extra amount of energy is required to break these noncovalent interactions before the peptide bond can be cleaved. This extra energy, called conformational stabilization energy and designated as $\Delta E_{B}$, is added to the Ea for each backbone cleavage (2). GB of basic side chains can be altered by salt bridges and other environmental factors. This change in GB of each basic residue is designated as $\triangle G B$ (3). Ea for side-chain neutral losses can also be affected by local conformation of the peptide. This change in Ea for each side-chain neutral loss is designated as $\Delta E_{S}$. To account for the discrepancies between predicted and experimental CID spectra, the above conformation-related parameters were included in the quantitative peptide fragmentation model for simulating the peptide CID spectrum. The values of these parameters were determined by maximizing the similarity between the predicted and experimental spectra as defined in the following equation [32]

$$
s=\frac{\sum \sqrt{I_{m}^{1} I_{m}^{2}}}{\sqrt{\left(\sum I_{m}^{1}\right)\left(\sum I_{m}^{2}\right)}}
$$

where $I_{m}^{1}$ and $I_{m}^{2}$ are intensities of an ion at $m / z=m$ for the two spectra. Two ions are considered the same ion if their $\mathrm{m} / \mathrm{z}$ ratios differ by less than $0.4 \mathrm{u}$.

All experimental spectra contain noise. An absolute best fit of the model to the experimental spectrum will generate a solution that contains large amount of noise, which may be interpreted as useful information. In this case, the model is over-fitted. To avoid over-fitting the model to the experimental spectrum, the dispersion of the values for the parameters $\Delta E_{B}, \Delta G B$, and $\Delta E_{S}$ is minimized at the same time when similarity between the predicted and the experimental spectra is maximized. In practice, the $Q$ values described in the following equation is maximized when determining the values of $\Delta E_{B}, \Delta G B$, and $\Delta E_{S}$.

$$
Q=s-\lambda d
$$

where $s$ is the similarity between the predicted spectrum and experimental spectrum defined in eq 1 , and $d$ is the dispersion of these conformation-related parameters as defined in eq 3 . The weight factor $\lambda$ is set as the largest value that does not change $s$ significantly when $Q$ is maximized. In this work, $\lambda$ is set at 0.002 , when the optimized value of $s$ typically decreases by less than 0.01 when compared to a $\lambda$ value of zero.

$$
\begin{aligned}
d= & \sqrt{\frac{\sum\left[\Delta E_{B i}-\left(\Delta E_{B i-1}+\Delta E_{B i+1}\right) / 2\right]^{2}}{N-1}} \\
& +\frac{1}{4} \sqrt{\frac{\sum \Delta E_{B}^{2}}{N-1}}+\sqrt{\frac{\sum \Delta G B^{2}}{N_{\text {Basic }}}}+\sqrt{\frac{\sum \Delta E_{S}^{2}}{N_{S}}}
\end{aligned}
$$

In eq $3, N$ represents the total number of residues in the peptide. NBasic represents the total number of basic sites in the peptide, including the $\mathrm{N}$-terminal amine and side chains of any arginine, histidine or lysine residues. $N_{S}$ stands for the total number of sites that may generate neutral losses, including the $\mathrm{N}$ - and C-termini as well as the side chains of aspartic acid, glutamic acid, serine, threonine, lysine, arginine, asparagine, and glutamine residues. The first term in eq 3 defines the deviation of $\Delta E_{B}$ of one site from its two neighbors. Minimizing $d$ ensures that the conformational stability map (the profile of $\Delta E_{B}$ versus residue number) is smooth and the magnitude of all conformation related parameters are reasonably small.

When optimizing $Q$ using Powell's direction set function optimization method [38], it was found that different starting points generate different solutions with similar optimized $Q$ values, indicating that more than one solution fit the experimental spectrum equally well. To resolve this problem, a simulated annealing method [39] was used to find 20 reasonably good solutions, followed by Powell's method to find the local maximum of $Q$ around each of the 20 solutions. The average $\Delta E_{B}$ values and their standard deviations were plotted against the residue number as the gas-phase conformational stability map. 


\section{Experimental}

Honey bee venom melittin, porcine pancreas glucagon, and human neuropeptide Y (Sigma, Saint Louis, MO) were separately dissolved in water to a concentration of $200 \mathrm{pmol} / \mu \mathrm{L}$, and analyzed on a Thermo Finnigan LTQ (San Jose, CA) linear ion trap mass spectrometer equipped with a static nanoelectrospray ionization source (Thermo) at a needle voltage of $1.0 \mathrm{kV}$ and heated capillary temperature of $200{ }^{\circ} \mathrm{C}$.

Tandem mass spectra of ions of interest were acquired with an activation time of $30 \mathrm{~ms}$, activation $q$ of 0.25 , isolation window of 3.0-4.0 u and normalized collision energy of $14-26 \%$. The collision energies were optimized to make the parent ions barely observable. Each spectrum was accumulated for three minutes in centroid mode.

To obtain large number of backbone cleavages, which are necessary for deducing maximal and reliable conformation-related parameters, the number of charges on a protonated peptide is preferably larger than the number of arginine residues in the peptide. When the number of charges is less or equal to the number of arginine residues in the peptide, these charges will all be sequestered on the basic arginine side chains and unable to move to the backbone to induce fragmentation [36, 40]. For maximal information, only those parent ions with charge states larger than the number of arginine residues in the peptide are included for the analysis.

\section{Results and Discussion}

\section{Gas-Phase Conformational Stability Map}

Triply charged melittin is used here as an example to illustrate how a gas-phase conformational stability map is derived. Figure 1 (top) shows the tandem spectrum of triply charged melittin. For triply charged melittin (GIGAVLKVLTTGLPALISWIKRKRQQ-NH2), two protons are sequestered by the two arginine side chains, leaving one proton freely movable across the backbone to cause fragmentation. Therefore, one expects to see strong b13 and/or y13 ions due to the labile L13-P14 cleavage. Indeed, strong b13 and y13 ions were predicted in the simulated tandem spectrum of melittin $(3+)$ as shown in Figure 1 (middle) [33]. However, little of these ions were observed in the experimental spectrum. Similarly, b16 and b17 ions were predicted to be present in the spectrum, but not seen in the experimental spectrum at all. On the other hand, the y15 ion (T11-G12 cleavage), which was predicted weak in the simulated spectrum, turned out to be strong in the experimental spectrum.

The discrepancies between the simulated spectrum and experimental spectrum are attributed to the conformation of the peptide, which is not modeled when predicting the tandem spectrum. The kinetic model describing the fragmentation process was modified by

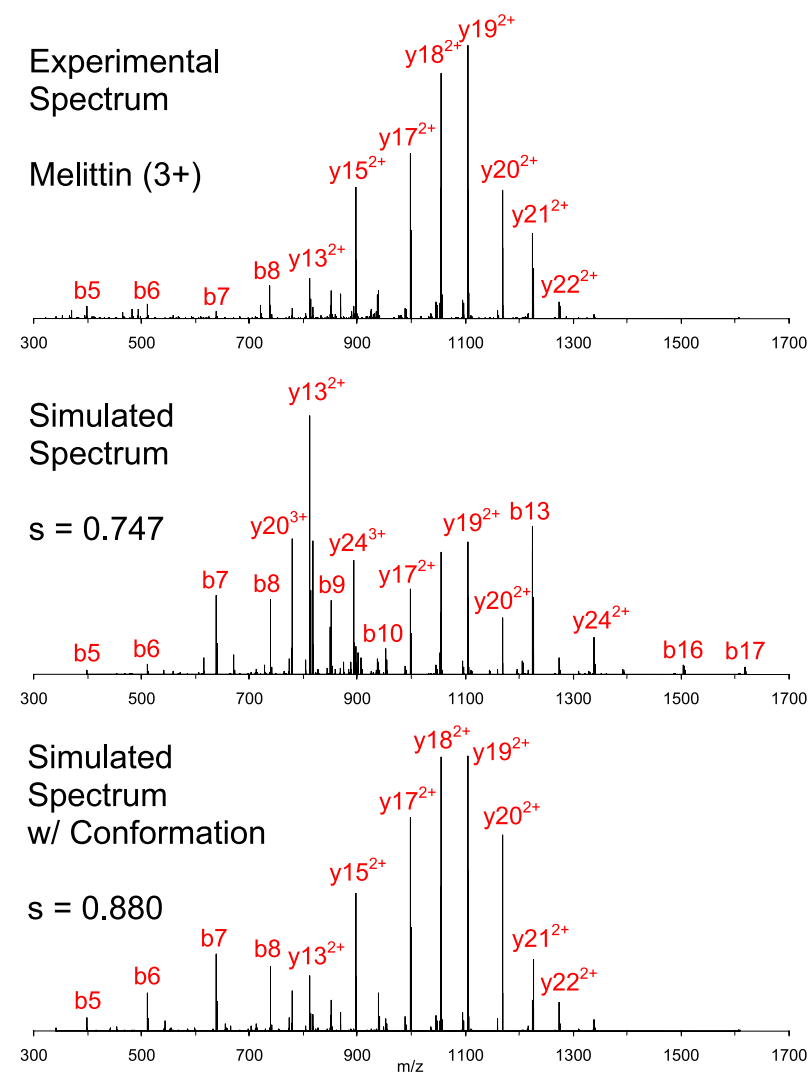

Figure 1. Comparison of experimental tandem spectrum of melittin $(3+)$ with its predicted spectra before and after considering the conformation effects. The predicted spectrum without considering the conformational effects (middle) is quite different from the experimental spectrum (top). After conformational effects are taken into account by optimizing the $\Delta E_{B}, \Delta G B$, and $\Delta E_{S}$ values, the predicted spectrum (bottom) is closely similar to the experimental spectrum.

adding conformation related parameters $\Delta E_{B}, \Delta G B$, and $\Delta E_{S}$ (see Computational Method section). These conformation-related parameters in the model were determined by maximizing $Q$ as shown in eq 2. Simulated annealing followed by function optimization was used to derive 20 solutions with optimized $Q$ values. After applying these optimized values of $\Delta E_{B}, \Delta G B$, and $\Delta E_{S}$ to the fragmentation model, the similarity score of the predicted spectrum increased from 0.747 to an average of 0.880 . Figure 1 (bottom) shows one of the predicted spectra after taking conformation effects into account. It can be seen that the predicted spectrum is now closely similar to the experimental spectrum.

Among the conformation-related parameters, $\Delta E_{B}$ values are of particular importance because every backbone peptide bond has a corresponding $\Delta E_{B}$ value, while only basic residues have $\triangle G B$ values and only residues with neutral losses have $\Delta E_{S}$ values. $\Delta G B$ values do provide some information, but the information is very limited due to limited number of basic residues in a peptide. It was found that the trend of $\Delta E_{S}$ values generally agrees with that of $\Delta E_{B}$ values. However, the $\Delta E_{S}$ curves are generally noisier than the $\Delta E_{B}$ 


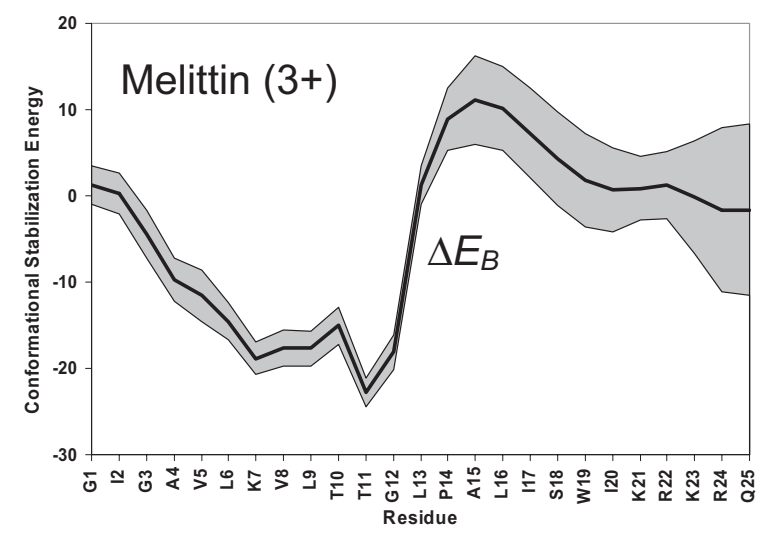

Figure 2. Gas-phase conformational stability map of melittin (3+).

curves, presumably because neutral-loss ions are usually weak and they are second-generation ions, which are more difficult to simulate. Because the $\Delta G B$ and $\Delta E_{S}$ values contain more noise and less information, they are not presented.

From the 20 sets of determined $\Delta E_{B}, \Delta G B$ and $\Delta E_{S}$ values, the $\Delta E_{B}$ values were plotted against the residue number to get a gas-phase conformational stability map. In a conformational stability map as shown in Figure 2, the solid curve represents the average $\Delta E_{B}$ values and the shaded area designates one standard deviation around the average $\Delta E_{B}$ value for each peptide bond. In Figure 2 and later, the peptide bond between residues $n$ and $n+1$ is represented as the residue $n$ in the horizontal axis. In a conformational stability map, a higher value of conformational stabilization energy $\Delta E_{B}$ indicates a more stable (or less flexible) local conformation and a lower value of $\Delta E_{B}$ indicates a less stable (or more flexible) local conformation. Large standard deviation (larger shaded area) indicates that the $\Delta E_{B}$ values cannot be determined decisively.

Since the fragmentation process is a competition of many pathways, changing the activation energies of all competing pathways by the same amount will change rate constants of all competing pathways by the same factor, and thus will not change the resulting predicted spectrum significantly. Therefore, when the $\Delta E_{B}$ values are calculated several times by simulated annealing/ function optimization without restricting the absolute values of the conformation related parameters (terms 2-4 in eq 3), conformational stability maps with similar shapes but different energy offsets will be obtained. Terms 2-4 in eq 3 are therefore applied to keep all conformation related parameters close to zero. As a result of minimizing the dispersion shown in eq 3 , the $\Delta E_{B}$ values in all conformational stability maps will be close to zero and their absolute values will have no physical meanings. Therefore, the maps show no overall stability of the peptide ions.

The stability map shown in Figure 2 indicates a flexible region around residues T11 and a stable region around residue L13-Q26, which explains the strong y15 ions and the absence of b13, b16, and b17 ions in the experimental spectrum.

\section{Gas-Phase Conformation of Melittin}

Melittin is a 26-residue peptide found in bee venom. In crystal [41,42] and some nonpolar environments [43] as well in multimeric form [44], melittin is an $\alpha$-helical peptide consisting of two $\alpha$-helices with a bend between residues Thr-11 and Gly-12. Molecular dynamics simulation $[45,46]$ also predicted two stable $\alpha$-helical regions and a flexible region around residues Thr-11 and Gly12. The high flexibility around residue Thr-11 is due in part to the presence of a proline at position 14, which results in a "missing" hydrogen bond in the largely $\alpha$-helical structure. The C-terminal helix is found more stable than the N-terminal helix by molecular dynamics simulation [46].

Figure 3 shows the gas-phase conformational stability maps of different charge states of melittin compared to its crystal structure [41]. Also included in the comparison are the crystallographic temperature factors of melittin. Temperature factors reflect the flexibility of atoms in the crystalline state [1]. Thus, the temperature factor versus residue number plot can be viewed as the conformational stability map in the crystalline state. A high-temperature factor indicates a more flexible region in the molecule. In Figure 3, the average temperature factor of the four backbone atoms (N, C- $\alpha, \mathrm{C}$, and $\mathrm{O}$ ) was used for each residue.

The temperature factors of melittin show a flexible region near residues Thr-11 and Gly-12. Comparison of the gas-phase conformational stability map to the stability map demonstrated in the temperature factors indicates correlations between the condensed-phase conformation and gas-phase conformation of $3+$ and $4+$ melittin ions. First, both $3+$ and $4+$ melittin ions show a flexible region near residues Thr-11 and Gly-12, which corresponds exactly to the flexible region determined by crystallography and other methods. Comparing to the flexible region near Thr-11 and Gly-12, the $\mathrm{N}$-terminal and C-terminal regions, which correspond to two $\alpha$-helices in the condensed phases, are more stable in the gas phase. While general correlation exists between the gas phase and condensed phases in terms of the flexible region and two $\alpha$-helical regions, the two $\alpha$-helical regions do not have equivalent stabilities in the gas phase. For $4+$ and especially $3+$ melittin ions, the C-terminal helix is found to be more stable than the $\mathrm{N}$-terminal helix in the gas phase. This is also true for mellitin in solution, as determined by molecular dynamics simulation [46]. In condensed phases, the two terminals of a peptide are usually more flexible (the "end effects"), as seen in the temperature factors in Figure 3. In gas phase, however, the stability on both terminals of the $3+$ and $4+$ melittin ions does not seem to decrease very much. Considering that $\alpha$-helices are primarily held together by hydrogen bonds, and the 

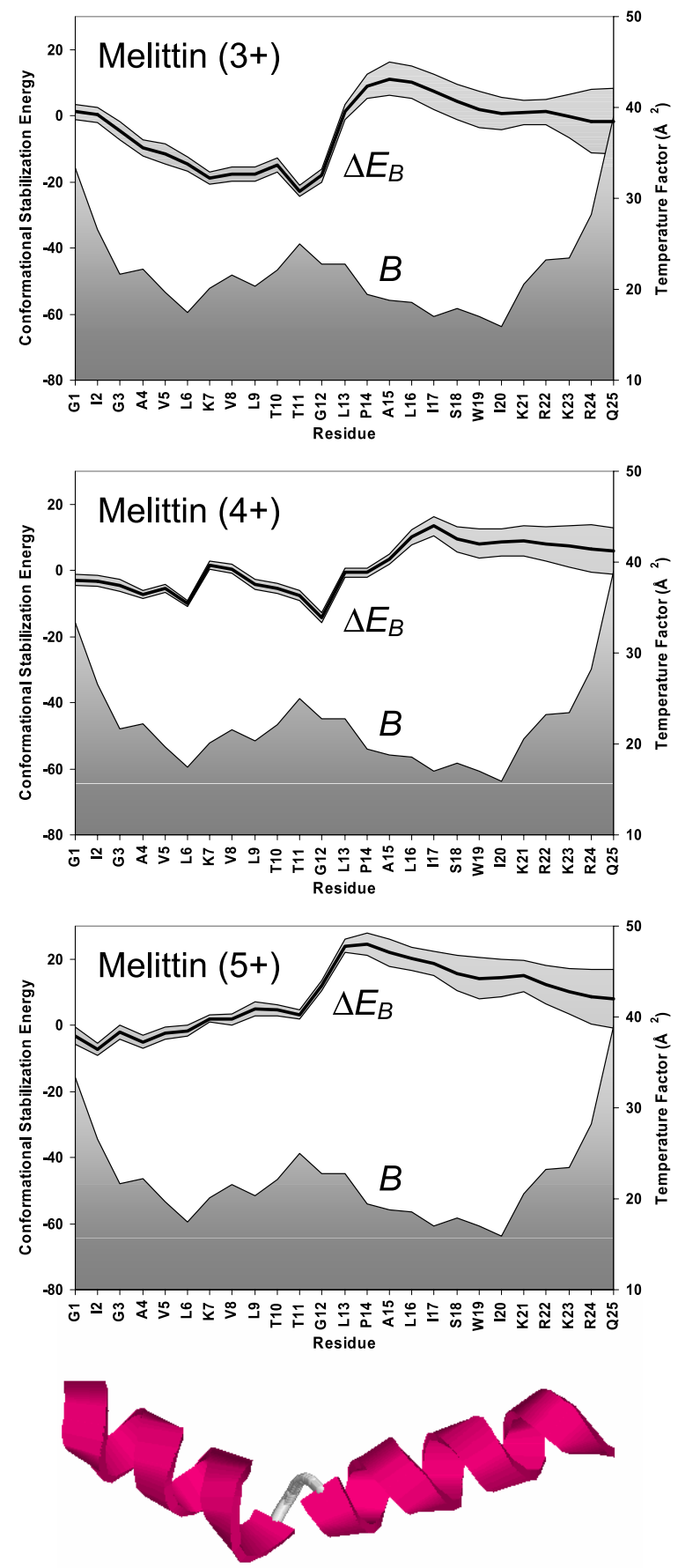

Figure 3. Conformational stability $\left(\Delta E_{B}\right)$ maps of different charge states of melittin compared to its crystal structure and temperature factors $(B)$.

strength of hydrogen bonds is about $5-6 \mathrm{kcal} / \mathrm{mol}$ in gas phase $[47,48]$, which is five-time stronger than in solution [48-50], it is reasonable to believe that the terminals of an $\alpha$-helix are more stable in the gas phase than in condensed phases. Other than the "end effects", the correlations between the stability maps in the condensed phases and gas phase for $3+$ and $4+$ melittin ions indicate that gaseous melittin ions up to four charges have a similar secondary structure as melittin in the condensed phases. This conclusion is consistent with the previous result that triply charged melittin retains its condensed phase secondary structure in the gas phase, as studied by intercharge repulsion measurement and molecular mechanics calculation [51]. For the $5+$ melittin ion, as seen in Figure 3, a major conformational change occurs as indicated by the flexible $\mathrm{N}$ terminal and C-terminal regions.

Note that it is not possible to assign secondary structures based on the gas-phase stability map alone. For example, the N-terminal region of the triply charged melittin may look too flexible to be an $\alpha$-helix. The authors merely like to point out the correlation between the stability maps in the gas phase and the condensed phase. In addition, because the absolute values of $\Delta E_{B}$ have no physical meanings, the overall up-shift of the map for the $5+$ melittin ions by no means suggests that the $5+$ ions are more stable than the $3+$ and $4+$ ions.

\section{Gas-Phase Conformation of Glucagon}

Glucagon is a polypeptide hormone consisting of 29 amino acid residues. The structure of porcine pancreas glucagon (HSQGTFTSDYSKYLDSRRAQDFVQWLMNT), as determined by X-ray crystallography [52], contains an $\alpha$-helix from residues Phe- 6 to Leu- 26 with the two terminus rather flexible. Figure 4 shows the gas-phase conformational stability maps of different charges of glucagon ions compared to its crystal structure. Temperature factors were not shown because they were not determined accurately due to the limited resolution $(3 \AA)$ of the determined crystal structure. The $\alpha$-helical region of the peptide is generally more stable in the gas phase than the flexible terminal regions for $3+$ and $4+$ ions. For the $5+$ glucagon ion, the peptide becomes flexible near Asp-15 and Ser-16, suggesting a disruption of the helical structure at higher charge states.

\section{Gas-Phase Conformation of Neuropeptide $Y$}

Neuropeptide $Y$ is a polypeptide with 36 residues and is the most abundant neuropeptide in mammalian central nervous system. The structure of human neuropeptide Y (YPSKPDNPGEDAPAEDMARYYSALRHYINLITRQRY- $\mathrm{NH}_{2}$ ) as determined by NMR in solution [53] contains a stable $\alpha$-helix at the C-terminal 24 residues (P13-Y36) and flexible N-terminal 12 residues.

Because neuropeptide $Y$ contains four arginine residues, the CID spectra of $3+$ and $4+$ ions of the peptide were not included in the analysis. Figure 5 shows the conformational stability maps of neuropeptide $\mathrm{Y}(5+)$ compared to its solution structure determined by NMR. The $\alpha$-helical region of the peptide is clearly more stable than the flexible $\mathrm{N}$-terminal region in the gas phase. In fact, no significant cleavages were observed between residues A18 and R35 in the CID spectra (not shown) of neuropeptide $\mathrm{Y}(5+)$, which by itself indicates secondary structure in this region. The large standard deviations in the C-terminal $\alpha$-helical region indicate that the 

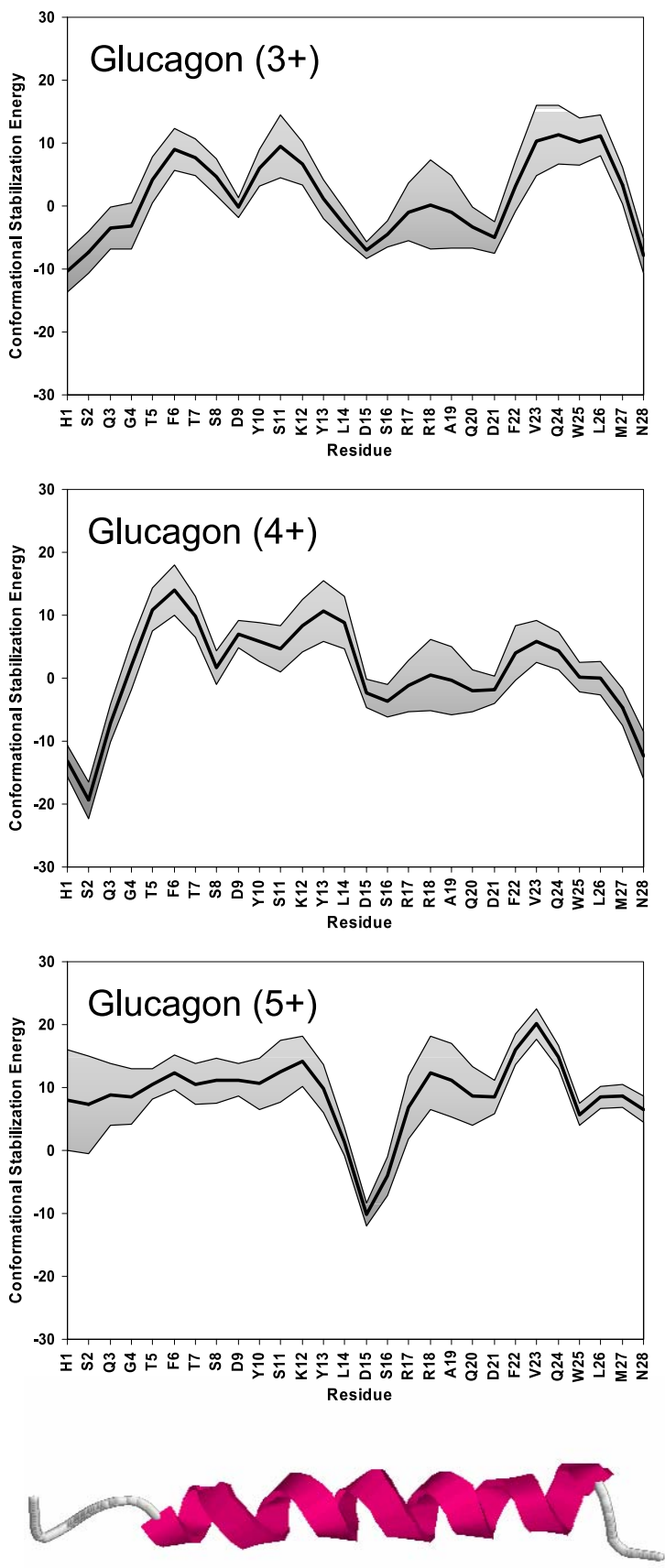

Figure 4. Conformational stability maps of different charge states of glucagon compared to its crystal structure.

$\Delta E_{B}$ values in this region cannot be determined decisively, due to the lack of fragment ions from cleavages in this region. However, the fact that even the lower part of the shaded area in the C-terminal region is significantly higher than the $\mathrm{N}$-terminal region indicates that the $\mathrm{C}$-terminal region is more stable than the $\mathrm{N}$-terminal region. The observation that the C-terminal $\alpha$-helical region is more stable than the $\mathrm{N}$-terminal region indicates that the C-terminal $\alpha$-helix of neuropeptide $\mathrm{Y}$ likely remains intact in the gas phase, and the N-terminal region remains flexible. It is noted that the three proline residues at sites 2, 5, and 8 are likely the reason for an unstable structure in the N-terminal region.

\section{Vacuum as a Nonpolar Environment}

In aqueous solution, the $\alpha$-helices in all three peptides are not stable when the peptides are in their monomeric forms [54-56]. These $\alpha$-helices, however, are stabilized by hydrophobic environments such as in methanol [43], chloroethanol [57], and trifluoroethanol [56, 58]. Part of the reason for stabilized $\alpha$-helices is that these nonpolar solvents stabilized the hydrogen bonds in the $\alpha$-helices. In gas phase, however, the $\alpha$-helices seem to be quite stable except at high charge states, as seen from the gas-phase stability maps. In the absence of solvent, intramolecular hydrogen bonds are much stronger because of the lack of competition from polar solvent molecules. In that respect, vacuum can be viewed as an ultimate nonpolar environment [51].

\section{Peptide Fragmentation as a Probe for Gas-Phase Conformation}

Melittin monomer contains as many as 20 hydrogen bonds according to its crystal structure [41, 42]. Considering that each hydrogen bond has a bond strength of about $5-6 \mathrm{kcal} / \mathrm{mol}$ in the gas phase [47, 48], breaking all these 20 hydrogen bonds requires more than 100 $\mathrm{kcal} / \mathrm{mol}$ of energy, which is far more than the energy require to break a backbone amide bond, which is about $40 \mathrm{kcal} / \mathrm{mol}[59,60]$. Therefore, it is logical to believe that only a few hydrogen bonds near the cleavage site
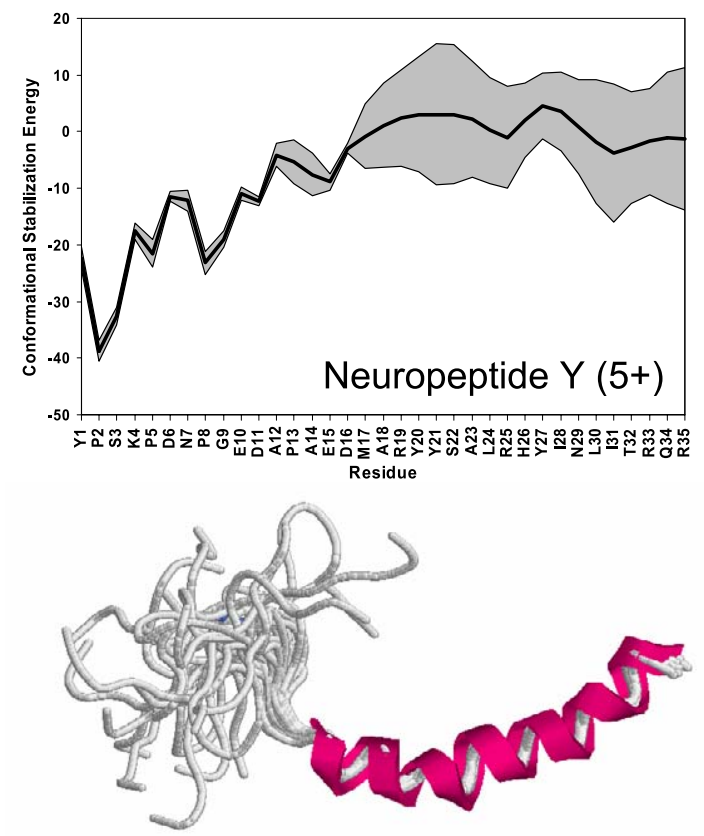

Figure 5. Conformational stability map of neuropeptide $\mathrm{Y}(5+)$ compared to its solution structure determined by NMR. 
are broken in the transition states during the backbone fragmentation, while the hydrogen bonds in the rest of the molecule remains similar to the ground state. The strong correlations of the gas-phase stability maps to the condensed-phase secondary structures of the three peptides demonstrated that this is indeed the case. The conformational stabilization energy described in the model reflects the amount of energy necessary to break those hydrogen bonds near the cleavage site.

Based on the above argument, it can be proposed that different fragmentation channels go through different transition states. Take the triply charged melittin for example; to form the y15 ions (cleavage between T11 and G12), only the hydrogen bonds near the flexible T11-G12 region need to be disrupted in the transitionstate, while the two $\alpha$-helices largely remain intact. A strong y15 ion is observed because little extra energy is required to break the hydrogen bonds near the flexible T11-G12 region. However, in the transition states to form b16 or b17 ions (L16-I17 and I17-S18 cleavages), the C-terminal $\alpha$-helix has to be disrupted, which takes much more energy. This explains why b16 and b17 ions are not observed in the experimental spectrum. The above argument, however, implies that the described technique may not be suitable to study conformation of small peptides. In a small peptide, the energy required to completely disrupt the noncovalent structure is small. Therefore, the conformation of its transition states may have no resemblance to its ground state.

For a large polypeptide such as a globular protein with extensive tertiary structure, care must be taken when interpreting the results. The presented model assumes that the rate-limiting step in a protein fragmentation is the cleavage of the covalent peptide bond. This may be true for a smaller peptide without much tertiary structure. For a protein with extensive tertiary structure, however, this may not be true because the separation of the two fragments after a bond cleavage may become the rate-limiting step due to strong longrange tertiary interactions. In that case, the conformational stabilization energy modeled here may not reflect the conformation stability near the cleavage site. Further investigations need to be conducted to determine which of the two processes is the rate-limiting step for globular proteins before the data obtained from the described technique can be appropriately interpreted. Of course, to generate any useful data for larger proteins, a high-resolution instrument such as a Fouriertransform ion-cyclotron resonance mass spectrometer is needed [31], with an appropriate fragmentation model developed.

A protein ion of the same charge state may have more than one conformation in the gas phase, as detected by gas-phase hydrogen/deuterium exchange $[22,24,26,61]$ and ion mobility study [62]. While Cassady and Carr found that for the $12+$ ubiquitin ions, the two conformations are clearly distinguishable in a CID experiment [24], Clemmer and coworkers reported that for ubiquitin ions with 8-10 charges, the CID spectra of a single charge state appear to be identical for different conformations, indicating that different conformers of each charge state rearrange to similar dissociation transition states before fragment formation [63]. This phenomenon suggests that "weak" noncovalent interactions contribute to the difference in the tertiary structure of ubiquitin, as opposed to the "strong" noncovalent interactions such as those hydrogen bonds in an $\alpha$-helix. These weak interactions cause protein ions of the same charge state to fold differently and exhibit different collisional cross-sections. These weak interactions are removed during the CID process, leaving only strong interactions in the transition-state. This hypothesis explains why ions of different collisional cross-sections have the same fragmentation spectra. The fact that the two different conformations in $12+$ ubiquitin ions are distinguishable by CID suggests that the difference between the two conformations involves some strong interactions. Collisional cross-section measurement, H/D exchange and recently used electron capture dissociation may reflect more of the most stable conformation of the protein because the protein ions are not heated, they do not distinguish strong interactions from weak interactions. CID reflects only the effects of strong interactions such as hydrogen bonds. The effects of weak interactions responsible for tertiary structures are often negligible in comparison. Therefore, CID provides information that is complimentary to most other techniques for studying protein gas-phase conformation.

A weakness of the presented CID technique is that it does not, by any means, provide information on the overall stability of the peptide ions. For example, it is unable to distinguish an all-helix peptide from an all-random coil peptide. However, as shown here, it is potentially very useful for detecting structural variations in peptide ions.

In the presented technique, the discrepancies between the experimental tandem spectrum of a peptide and its theoretical spectrum predicted from the peptide fragmentation model are attributed to the secondary/ tertiary structure of the peptide. Apparently, these discrepancies may also be caused by imperfections in the fragmentation model. For example, it is potentially possible that the extremely flexible region detected near Asp-15 of glucagons 5+ (Figure 4) is an artifact caused by imperfection in the model for highly charged ions. Ruling out this possibility is difficult due to the lack of conformational "gold standards" of gaseous peptides. To serve as a "gold standard" for this technique the peptide ions must have significant structural variations inside them and must have detailed structural information available for each individual residue. However, the strong correlations between the gas-phase conformational stability maps of lower-charged peptides and their condensed-phase secondary structures demonstrate that the effect of imperfections in the model is small, at least for these lower-charged peptide ions. More work need to be done in understanding the 
mechanisms of peptide fragmentation to minimize the imperfections in the fragmentation model. At this point, the site-specific information of gaseous peptide/protein ions will certainly contribute to our understanding of structure of gaseous peptides/proteins.

\section{Conclusions}

Protein CID spectrum, combined with fragmentation modeling, was used to quantitatively access site-specific information on conformational stabilities of gaseous peptide ions. The gas-phase conformational stability maps of several $\alpha$-helical peptides as determined by this technique correlate closely with their condensed-phase secondary structures, suggesting similarities between the gas-phase and condensed-phase conformations of these $\alpha$-helical peptides. The strong correlations between gas-phase and condensed-phase stability maps also demonstrate that low-energy CID process does not provide sufficient energy to completely disrupt the structures of these peptides, as well as the validity of the presented technique for studying peptide gas-phase conformations. The site-specific stability map described here is complementary to the data obtained by more conventional techniques in that only strong noncovalent interactions are monitored. Combining site-specific stability map with other techniques such as collisional cross-section measurement, H/D exchange and molecular modeling it is potentially possible to obtain a much detailed picture of conformation and dynamics of gaseous peptides.

\section{Acknowledgments}

The authors are grateful to Bela Paizs of German Cancer Research Center and Hai Pan of Amgen for helpful discussions during preparation of the manuscript.

\section{References}

1. Petsko, G. A.; Ringe, D. Fluctuations in protein structure from X-ray diffraction. Annu. Rev. Biophys. Bioeng. 1984, 13, 331-371.

2. Bax, A. Two-dimensional NMR and protein structure. Annu. Rev. Biochem. 1989, 58, 223-256.

3. Wuthrich, K. Protein structure determination in solution by NMR spectroscopy. J. Biol. Chem. 1990, 265, 22059-22062.

4. Clore, G. M.; Gronenborn, A. M. Two-, three-, and four-dimensional NMR methods for obtaining larger and more precise three-dimensional structures of proteins in solution. Annu. Rev. Biophys. Biophys. Chem. 1991, 20, 29-63.

5. Kelly, S. M.; Price, N. C. The application of circular dichroism to studies of protein folding and unfolding. Biochim. Biophys. Acta. 1997, 1338, 161-185

6. Li, R.; Woodward, C. The hydrogen exchange core and protein folding. Protein Sci. 1999, 8, 1571-1591.

7. Englander, S. W. Protein folding intermediates and pathways studied by hydrogen exchange. Annu. Rev. Biophys. Biomol. Struct. 2000, 29, 213-238.

8. Zhang, Z.; Smith, D. L. Determination of amide hydrogen exchange by mass spectrometry: A new tool for protein structure elucidation. Protein Sci. 1993, 2, 522-531.

9. Smith, D. L.; Deng, Y.; Zhang, Z. Probing the noncovalent structure of proteins by amide hydrogen exchange and mass spectrometry. J. Mass Spectrom. 1997, 32, 135-146.

10. Engen, J. R.; Smith, D. L. Investigating protein structure and dynamics by hydrogen exchange MS. Anal. Chem. 2001, 73, 256A-265A.

11. Kaltashov, I. A. Probing protein dynamics and function under native and mildly denaturing conditions with hydrogen exchange and mass spectrometry. Int. J. Mass Spectrom. 2005, 240, 249-259.
12. Hoaglund-Hyzer, C. S.; Counterman, A. E.; Clemmer, D. E. Anhydrous protein ions. Chem. Rev. 1999, 99, 3037-3079.

13. Jarrold, M. F. Peptides and proteins in the vapor phase. Annu. Rev. Phys. Chem. 2000, 51, 179-207.

14. Covey, T.; Douglas, D. J. Collision cross sections for protein ions. J. Am. Soc. Mass Spectrom. 1993, 4, 616-623.

15. Chen, Y. L.; Campbell, J. M.; Collings, B. A.; Konermann, L.; Douglas, D. J. Stability of a highly charged noncovalent complex in the gas phase-holomyoglobin. Rapid Commun. Mass Spectrom. 1998, 12, 10031010 .

16. Gill, A. C.; Jennings, K. R.; Wyttenbach, T.; Bowers, M. T. Conformations of biopolymers in the gas phase: A new mass spectrometric method. Int. J. Mass Spectrom. 2000, 196, 685-697.

17. Counterman, A. E.; Clemmer, D. E. Large anhydrous polyalanine ions: Evidence for extended helices and onset of a more compact state. J. Am. Chem. Soc. 2001, 123, 1490-1498.

18. Purves, R. W.; Barnett, D. A.; Ells, B.; Guevremont, R. Elongated conformers of charge states +11 to +15 of bovine ubiquitin studied using ESI-FAIMS-MS. J. Am. Soc. Mass Spectrom. 2001, 12, 894-901.

19. Wyttenbach, T.; Kemper, P. R.; Bowers, M. T. Design of a new electrospray ion mobility mass spectrometer. Int. J. Mass Spectrom. 2001, 212, 13-23.

20. Wyttenbach, T.; Bowers, M. T. Gas-phase conformations: The ion mobility/ion chromatography method. Mod. Mass Spectrom. 2003, 225, 207-232.

21. Thalassinos, K.; Slade, S. E.; Jennings, K. R.; Scrivens, J. H.; Giles, K.; Wildgoose, J.; Hoyes, J.; Bateman, R. H.; Bowers, M. T. Ion mobility mass spectrometry of proteins in a modified commercial mass spectrometer. Int. J. Mass Spectrom. 2004, 236, 55-63.

22. Suckau, D.; Shi, Y.; Beu, S. C.; Senko, M. W.; Quinn, J. P.; Wampler, F. M., III; McLafferty, F. W. Coexisting stable conformations of gaseous protein ions. Proc. Natl. Acad. Sci. U.S.A. 1993, 90, 790-793.

23. Wood, T. D.; Chorush, R. A.; Wampler, F. M.; Little, D. P.; Oconnor, P. B.; McLafferty, F. W. Gas-phase folding and unfolding of cytochrome c cations. Proc. Natl. Acad. Sci. U.S.A. 1995, 92, 2451-2454.

24. Cassady, C. J.; Carr, S. R. Elucidation of isomeric structures for ubiquitin. J. Mass Spectrom. 1996, 31, 247-254.

25. Freitas, M. A.; Hendrickson, C. L.; Emmett, M. R.; Marshall, A. G. High-field Fourier transform ion cyclotron resonance mass spectrometry for simultaneous trapping and gas-phase hydrogen/deuterium exchange of peptide ions. I. Am. Soc. Mass Spectrom. 1998, 9, 1012-1019.

26. Freitas, M. A.; Hendrickson, C. L.; Emmett, M. R.; Marshall, A. G. Gas-phase bovine ubiquitin cation conformations resolved by gas-phase hydrogen/deuterium exchange rate and extent. Int. J. Mass Spectrom. 1999, 185/186/187, 565-575.

27. Wyttenbach, T.; Bowers, M. T. Gas phase conformations of biological molecules: The hydrogen/deuterium exchange mechanism. J. Am. Soc. Mass Spectrom. 1999, 10, 9-14.

28. Horn, D. M.; Breuker, K.; Frank, A. J; McLafferty, F. W. Kinetic intermediates in the folding of gaseous protein ions characterized by electron capture dissociation mass spectrometry. J. Am. Chem. Soc. 2001, 123, 9792-9799.

29. Breuker, K.; Oh, H.; Horn, D. M.; Cerda, B. A.; McLafferty, F. W. Detailed unfolding and folding of gaseous ubiquitin ions characterized by electron capture dissociation. J. Am. Chem. Soc. 2002, 124, 6407-6420.

30. Oh, H.; Breuker, K.; Sze, S. K.; Ge, Y.; Carpenter, B. K.; McLafferty, F. W. Secondary and tertiary structures of gaseous protein ions characterized by electron capture dissociation mass spectrometry and photofragment spectroscopy. Proc. Natl. Acad. Sci. U.S.A. 2002, 99, 15863-15868.

31. Wu, Q. Y.; Vanorden, S.; Cheng, X. H.; Bakhtiar, R.; Smith, R. D. Characterization of cytochrome $c$ variants with high-resolution FTICR mass spectrometry-correlation of fragmentation and structure. Anal. Chem. 1995, 67, 2498-2509.

32. Zhang, Z. Prediction of low-energy collision-induced dissociation spectra of peptides. Anal. Chem. 2004, 76, 3908-3922.

33. Zhang, Z. Prediction of low-energy collision-induced dissociation spectra of peptides with three or more charges. Anal. Chem. 2005, 77, 6364-6373.

34. Zhang, Z. De novo peptide sequencing based on a divide-and-conquer algorithm and peptide tandem spectrum simulation. Anal. Chem. 2004, $76,6374-6383$

35. Schlosser, A.; Lehmann, W. D. Special feature: Commentary-fivemembered ring formation in unimolecular reactions of peptides: A key structural element controlling low-energy collision-induced dissociation of peptides. J. Mass Spectrom. 2000, 35, 1382-1390.

36. Wysocki, V. H.; Tsaprailis, G.; Smith, L. L.; Breci, L. A. Special feature: Commentary-mobile and localized protons: A framework for understanding peptide dissociation. J. Mass Spectrom. 2000, 35, 1399-1406.

37. Paizs, B.; Suhai, S. Fragmentation pathways of protonated peptides. Mass Spectrom. Rev. 2005, 24, 508-548.

38. Price, W. D.; Schnier, P. D.; Williams, E. R. Tandem mass spectrometry of large biomolecule ions by blackbody infrared radiative dissociation. Anal. Chem. 1996, 68, 859-866.

39. Kirkpatrick, S.; Gelatt, C. D. J.; Vecchi, M. P. Optimization by simulated annealing. Science 1983, 220, 671-680.

40. Dongre, A. R.; Jones, J. L.; Somogyi, A.; Wysocki, V. H. Influence of peptide composition, gas-phase basicity, and chemical modification on fragmentation efficiency-evidence for the mobile proton model. J. Am. Chem. Soc. 1996, 118, 8365-8374. 
41. Terwilliger, T. C.; Eisenberg, D. The structure of melittin. I. Structure determination and partial refinement. J. Biol. Chem. 1982, 257, 60106015.

42. Terwilliger, T. C.; Eisenberg, D. The structure of melittin. II. Interpretation of the structure. J. Biol. Chem. 1982, 257, 6016-6022.

43. Bazzo, R.; Tappin, M. J.; Pastore, A.; Harvey, T. S.; Carver, J. A.; Campbell, I. D. The structure of melittin. A ${ }^{1} \mathrm{H}-\mathrm{NMR}$ study in methanol. Eur. J. Biochem. 1988, 173, 139-146

44. Brown, L. R.; Lauterwein, J.; Wuthrich, K. High-resolution ${ }^{1} \mathrm{H}-\mathrm{NMR}$ studies of self-aggregation of melittin in aqueous solution. Biochim. Biophys. Acta 1980, 622, 231-244

45. Pastore, A.; Harvey, T. S.; Dempsey, C. E.; Campbell, I. D. The dynamic properties of melittin in solution. Investigations by NMR and molecular dynamics. Eur. Biophys. J. 1989, 16, 363-367

46. Sessions, R. B.; Gibbs, N.; Dempsey, C. E. Hydrogen bonding in helical polypeptides from molecular dynamics simulations and amide hydrogen exchange analysis: Alamethicin and melittin in methanol. Biophys. J. 1998, 74, 138-152.

47. Mitchell, J. B. O.; Price, S. L. The nature of the N HOC hydrogen bond: An intermolecular perturbation theory study of the formamide/formaldehyde complex. J. Comp. Chem. 1990, 11, 1217-1233.

48. Ben-Tal, N.; Sitkoff, D.; Topol, I. A.; Yang, A. S.; Burt, S. K.; Honig, B. Free energy of amide hydrogen bond formation in vacuum, in water, and in liquid alkane solution. J. Phys. Chem. B 1997, 101, 450-457.

49. Scholtz, J. M.; Qian, H.; York, E. J.; Stewart, J. M.; Baldwin, R. L. Parameters of helix-coil transition theory for alanine-based peptides of varying chain lengths in water. Biopolymers 1991, 31, 1463-1470.

50. Scholtz, J. M.; Marqusee, S.; Baldwin, R. L.; York, E. J.; Stewart, J. M.; Santoro, M.; Bolen, D. W. Calorimetric determination of the enthalpy change for the $\alpha$-helix to coil transition of an alanine peptide in water. Proc. Natl. Acad. Sci. U.S.A. 1991, 88, 2854-2858.

51. Kaltashov, I. A.; Fenselau, C. Stability of secondary structural elements in a solvent-free environment-the $\alpha$ helix. Proteins 1997, 27, 165-170.

52. Sasaki, K.; Dockerill, S.; Adamiak, D. A.; Tickle, I. J.; Blundell, T. X-ray analysis of glucagon and its relationship to receptor binding. Nature 1975, 257, 751-757.
53. Monks, S. A.; Karagianis, G.; Howlett, G. J.; Norton, R. S. Solution structure of human neuropeptide Y. J. Biomol. NMR 1996, 8, 379-390.

54. Lauterwein, J.; Brown, L. R.; Wuthrich, K. High-resolution ${ }^{1} \mathrm{H}-\mathrm{NMR}$ studies of monomeric melittin in aqueous solution. Biochim. Biophys. Acta 1980, 622, 219-230

55. Boesch, C.; Bundi, A.; Oppliger, M.; Wuthrich, K. ${ }^{1} \mathrm{H}$ nuclear-magneticresonance studies of the molecular conformation of monomeric glucagon in aqueous solution. Eur. J. Biochem. 1978, 91, 209-214

56. Nordmann, A.; Blommers, M. J. J.; Fretz, H.; Arvinte, T.; Drake, A. F. Aspects of the molecular structure and dynamics of neuropeptide Y. Eur. J. Biochem. 1999, 261, 216-226.

57. Gratzer, W. B.; Beaven, G. H.; Rattle, H. W.; Bradbury, E. M. A conformational study of glucagon. Eur. J. Biochem. 1968, 3, 276-283

58. Mierke, D. F.; Durr, H.; Kessler, H.; Jung, G. Neuropeptide Y: Optimized solid-phase synthesis and conformational analysis in trifluoroethanol. Eur. J. Biochem. 1992, 206, 39-48

59. Vekey, K.; Somogyi, A.; Wysocki, V. H. Average activation energies of low-energy fragmentation processes of protonated peptides determined by a new approach. Rapid Commun. Mass Spectrom. 1996, 10, 911-918.

60. Klassen, J. S.; Kebarle, P. Collision-induced dissociation threshold energies of protonated glycine, glycinamide, and some related small peptides and peptide amino amides. J. Am. Chem. Soc. 1997, 119, 6552-6563.

61. Wang, F.; Freitas, M. A.; Marshall, A. G.; Sykes, B. D. Gas-phase memory of solution-phase protein conformation: H/D exchange and Fourier transform ion cyclotron resonance mass spectrometry of the $\mathrm{N}$-terminal domain of cardiac troponin C. Int. J. Mass Spectrom. 1999, 192, 319-325.

62. Purves, R. W.; Barnett, D. A.; Guevremont, R. Separation of protein conformers using electrospray-high field asymmetric waveform ion mobility spectrometry-mass spectrometry. Int. J. Mass Spectrom. 2000, 197, 163-177.

63. Badman, E. R.; Hoaglund-Hyzer, C. S.; Clemmer, D. E. Dissociation of different conformations of ubiquitin ions. J. Am. Soc. Mass Spectrom. 2002, 13, 719-723. 